University of Nebraska - Lincoln

DigitalCommons@University of Nebraska - Lincoln

Faculty Publications in Computer \& Electronics Electrical \& Computer Engineering, Department Engineering (to 2015)

2005

\title{
Combined Asterism and Sphere decoder for large Asymmetric MIMO systems
}

\author{
Phillip Conder \\ University of Wollongong \\ Tadeusz Wysocki \\ University of Nebraska-Lincoln, wysocki@uow.edu.au
}

Follow this and additional works at: https://digitalcommons.unl.edu/computerelectronicfacpub

Part of the Computer Engineering Commons

Conder, Phillip and Wysocki, Tadeusz, "Combined Asterism and Sphere decoder for large Asymmetric MIMO systems" (2005). Faculty Publications in Computer \& Electronics Engineering (to 2015). 17. https://digitalcommons.unl.edu/computerelectronicfacpub/17

This Article is brought to you for free and open access by the Electrical \& Computer Engineering, Department of at DigitalCommons@University of Nebraska - Lincoln. It has been accepted for inclusion in Faculty Publications in Computer \& Electronics Engineering (to 2015) by an authorized administrator of DigitalCommons@University of Nebraska - Lincoln. 


\title{
Combined Asterism and Sphere decoder for large Asymmetric MIMO systems
}

\author{
Phillip Conder, Tadeusz A.Wysocki \\ Telecommunications and Information Technology Research Institute \\ University of Wollongong, Wollongong Australia \\ Email: \{pc20,wysocki\}@uow.edu.au
}

\begin{abstract}
The area of Multiple Input Multiple Output (MIMO) communications systems has received enormous attention recently as they can provide a roughly linear increase in data rate by using Multiple Transmit and Receive antennas. The optimal detection strategy for a MIMO receiver is to perform a Maximum-Likelihood (ML) search over all possible transmitted symbol combinations which has an exponential complexity when the constellation size of number of transmit antennas increase.

Sphere and Asterism decoders are schemes that achieved ML performance for MIMO systems, but both have shortcomings in computational complexity, Sphere decoding when $n_{t}>n_{r}$ and Asterism when $n_{t}<n_{r}$. This paper combines Asterism and Sphere decoding for Asymmetric MIMO systems to reduce the overall computational complexity for any number of antennas.
\end{abstract}

\section{INTRODUCTION}

In recent years the employment of multi antenna arrays at both transmit and receive sites has received much interest because it is capable of significantly increase the theoretical capacity of wireless communications systems, in particular in the area of Multiple In Multiple Out (MIMO) systems [1]. At the same time as increased data rates, future mobile communications are promised to be small, lightweight mobile devices which present problems to the implementation of multiple antenna systems.

It was shown in [2], that using such a ML decoder with less receive than transmit antennas could still provide sufficient increase in data rate, hence removing the need for additional receive antennas on a receiving device, such as a mobile terminal. Thus reducing the size and cost of mobile terminals by reducing the number receive antennas may be a factor in the implementation of MIMO especially for systems with a larger number of transmit antennas.

Sub-optimal decoding schemes such as Zero Forcing (ZF) and the Bell Labs Layered Space-Time (BLAST)[3] are low complexity algorithms that perform best when the number of receive antennas is greater than the number of transmit antennas, but perform poorly when antenna numbers are equal. While near optimal Sphere or lattice decoders [4][6], provide linear decoding only at high $S N R$ and when the number of receive antennas is at least equal to the number of transmit antennas and are computationally burdensome when the number of transmit is larger than the number of receive antennas.
Asterism decoding proposed in [5], described a scheme that achieved ML performance for MIMO systems. By considering the larger complex constellation created by a multiple transmit antennas and a single receive antenna. The decoder design was then extended to achieve ML like performance for any number of receive antennas. Although Asterism decoding's computational complexity is an order of magnitude less that ML decoding, it is related to the number of transmit antennas and constellation size and making it far too complex for systems with a large number of transmit antennas.

The paper is ordered as follows: Section 2 gives a brief system description of MIMO system and decoders such as Maximum Likelihood, Zero Forcing (ZF) for systems where $n_{t}>n_{r}$. Section 3 reviews Sphere decoding Asterism decoding for MIMO systems. While Section 4 describes how Asterism decoding can be used in conjunction with Sphere decoding to reduce the computational complexity of systems with $n_{t}>n_{r}$. Finally Section 5 presents simulations results of performance and complexity of the combined Asterism and Sphere decoder.

\section{Multiple In Multiple Out Systems}

The Multiple In Multiple out approach was first introduced by Lucent's Bell Labs, with their BLAST family of Space Time Code structures [3]. An uncoded Vertical Bell Laboratories Layered Space-Time (VBLAST) scheme, where the input bit stream is de-multiplexed into $n_{t}$ substreams, is considered in this paper. Let $n_{t}$ be the number of transmit and $n_{r}$ be the number of receive antennas, and $s=\left(s_{1}, s_{2}, \ldots s_{n_{t}}\right)^{T}$ denote the vector of symbols of constellation size $C$, transmitted in one symbol period. The received vector $R=\left(R_{1}, R_{2} \ldots R_{n_{r}}\right)^{T}$ :

$$
R=H s+n
$$

where $n=\left(n_{1}, n_{2}, \ldots n_{n_{r}}\right)^{T}$ is the noise vector of additive white Gaussian noise of variance $\sigma^{2}$ equal to $\frac{1}{2}$ per dimension. The $n_{r} \times n_{t}$ channel matrix:

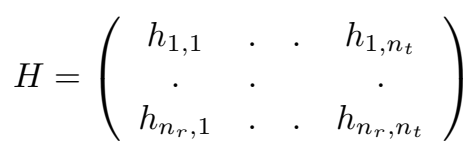

contains independent identical distribution (i.i.d.) complex fading gains $h_{i, j}$ from the $j^{\text {th }}$ transmit antenna to the $i^{\text {th }}$ 
receive antenna. We assume Rayleigh flat fading where the magnitude of the elements of $H$ have a Rayleigh distribution.

Optimal Maximum Likelihood decoding is achieved by minimising

$$
\|H s-R\|^{2}
$$

for all elements of $s$, which are symbols of constellation of size $C$. This would produce a search of length $C^{n_{t}}$, which for a large MIMO system using 8 transmit antennas and a relatively simple modulation of $Q P S K$ gives 65536 possibilities that need to be tested by (3).

\section{A. Zero Forcing and VBLAST}

Zero Forcing (or nulling) uses a pseudo inverse of $H$ to produce estimates, $\widetilde{s}$, of the transmitted symbols. The VBLAST [3] algorithm detects each symbol of $\widetilde{s}$ one at a time and considers the remaining symbols as interferers. For example, when detecting the first symbol there are no previously detected symbols to cancel out so detection is the same as ZF algorithm, for the second detected symbol the first detected symbols is canceled out of the received vector. This modified received vector has fewer interferers and better performance due to a higher level of diversity. This methods of successive cancelation is continued until all $n_{t}$ symbols are detected. Obviously an incorrect symbol selection in the early stages will create errors in the following stages, this called error propagation.

Calculation of $\widetilde{s}$ becomes difficult when $n_{t}>n_{r}$ as a square matrix is needed for the pseudo inverse calculation. The channel matrix can be made square by padding $H$ into a square matrix with zeroes and very small diagonal entries:

$$
H_{\text {pad }}=\left(\begin{array}{cccc}
h_{1,1} & h_{1,2} & h_{1,3} & h_{1,4} \\
h_{2,1} & h_{2,2} & h_{2,3} & h_{2,4} \\
0 & 0 & \gamma & 0 \\
0 & 0 & 0 & \gamma
\end{array}\right)
$$

where $\gamma$ is a small magnitude constant, we use $\gamma=10^{-3}$ in our simulations. By adding some extra zeros in the received vector $\widetilde{s_{i}}$ can be calculated:

$$
\tilde{s}=H_{p a d}^{-1}\left(\begin{array}{c}
R \\
0 \\
0
\end{array}\right)
$$

Due to the fact that the number of interferers is more than the number of equations error propagation is more prevalent, making ZF and VBLAST decoders unsuitable for $n_{t}>n_{r}$ systems.

\section{SPHERE DECODING}

The principle of Sphere decoding is to search the closest lattice point to the received vector within a sphere of radius $D$ centered at $\widetilde{s}$. The Sphere decoder of [4] decomposes the channel matrix and received vector from complex numbers into its real and imaginary parts to produce a channel matrix of twice the size of the original. The multi-dimensional sphere now becomes an interval centered around $\widetilde{s}$.
The Cholesky factorisation of $H$ becomes:

$$
Q=\left(\begin{array}{cccc}
q_{1,1} & q_{1,2} & q_{1,3} & q_{1,4} \\
0 & q_{1,2} & q_{2,3} & q_{2,4} \\
0 & 0 & q_{3,3} & q_{3,4} \\
0 & 0 & 0 & q_{4,4}
\end{array}\right)
$$

and is used to determine the radius size used at each stage based on the starting radius and any previously decoded symbols. The first sphere radius becomes:

$$
D_{\text {first }}=\frac{D}{q_{4,4}}
$$

The choice of stating radius $D$ is very crucial to the speed of the algorithm. A radius too small will contain no lattice points causing the decoder to restart with a larger radius, while a radius too large will contain too many points.

\section{A. Sphere decoding for $n_{t}>n_{r}$}

The Sphere decoder is essentially the same for $n_{t}>n_{r}$ but uses the Padded channel matrix $H_{\text {pad }}$ to not only determine $\widetilde{s}$ but also the Cholesky factorisation. While the padding of $H$ is satisfactory for the calculation of $\widetilde{s}$, when used for the Cholesky factorisation it produces very small numbers for the lowest $n_{t}-n_{r}$ levels of the triangular matrix. When these very small values of $q_{i, i}$ are used in (7) the radius becomes $D_{\text {first }}$ becomes very large. This means that all constellation points are found inside the sphere and that the Sphere decoder has a complexity of the order of $C^{n_{t}-n_{r}}$. Sphere decoder decomposes to the complexity of ML detection when $n_{r}=1$. This is illustrated in Figure 1 which show the complexity of the Sphere decoder algorithm for $n_{t}=8$ and various number of receive antennas.

The complexity of the Sphere decoder when $n_{r}=4$ is approximately $C$ times more complex than for $n_{r}=5$ which in turn is approximately $C$ times more complex than $n_{r}=6$.

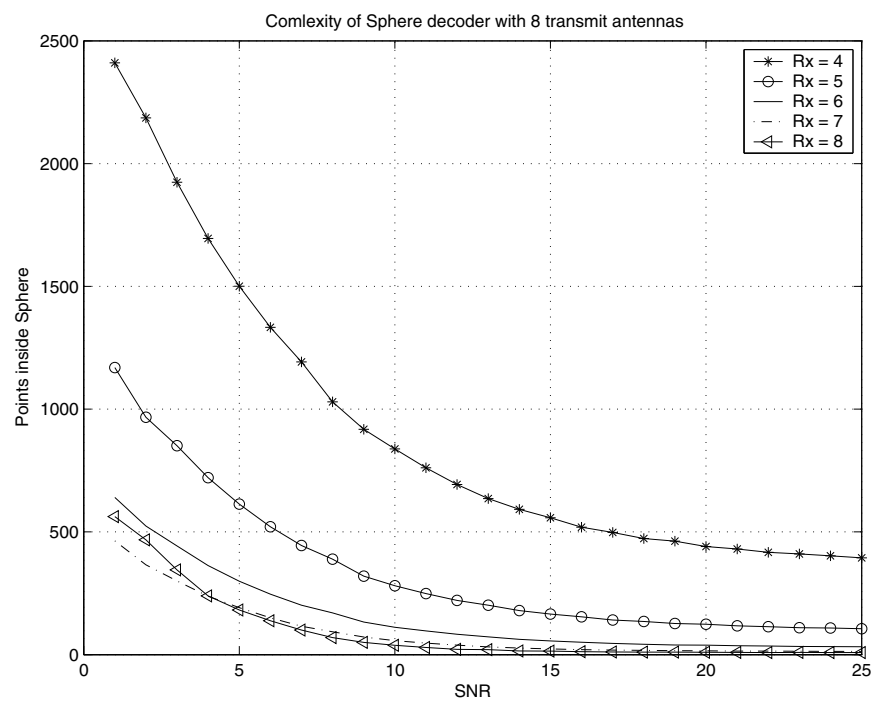

Fig. 1. The complexity of Sphere decoder versus $S N R$ for $n_{t}=8$ using $Q P S K$ and $n_{r}=4,5,6,7,8$. 


\section{Asterism DeCODING}

Asterism decoding [5] was created to reduce the computational complexity of Maximum Likelihood decoding and yet retain the performance and flexibility of reducing the number of receive antennas. By considering the larger complex constellation created by a multiple transmit antennas and a single receive antenna.

The complex constellation generated by equation (3) can be divided into $C$ smaller groups or Asterisms as shown in Figure 2. Each of these Asterisms can in turn be divided into $C$ smaller Asterisms, and so on for $n_{t}$.

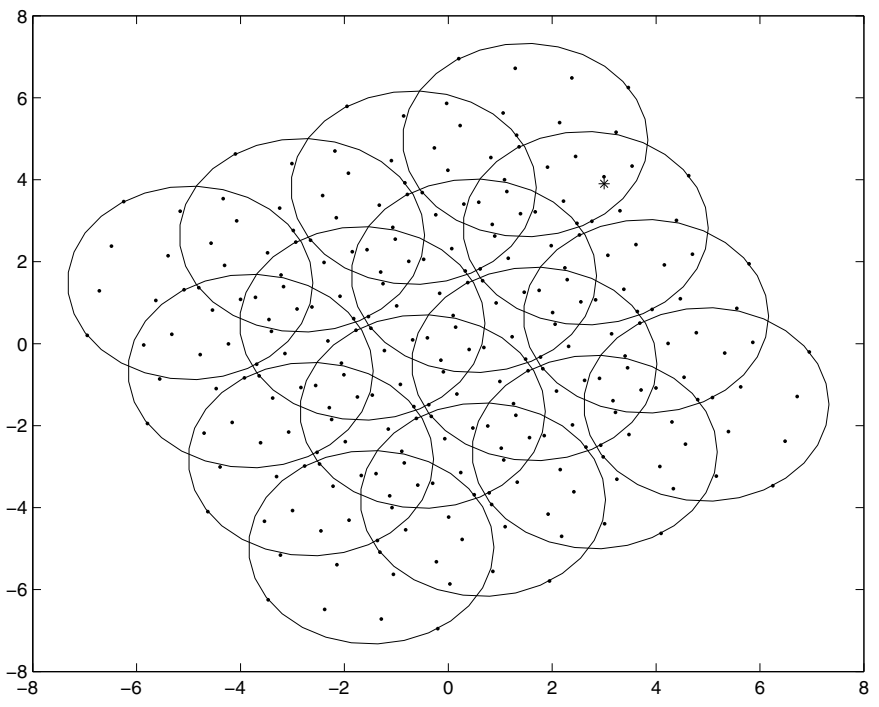

Fig. 2. Complex constellation from (3) grouped into C Asterisms.

Finding the ML solution without having to test every point by grouping the complex constellation into Asterisms is the main concept behind the Asterism decoding.

For ease of explanation, we make the assumption that the magnitude of $H$ in (3) is decreasing i.e. $\left|h_{1}\right|$ is the largest and $\left|h_{3}\right|$ is the smallest. The radius of the Asterism at detection stage $k$ is:

$$
\operatorname{Radius}_{(k)}=\beta \times \sum_{j=k+1}^{n_{t}}|h(j)|
$$

where $\beta=$ largest symbol magnitude, which for 16QAM is $\sqrt{18}$ the magnitude for the symbols $[3+3 i,-3+3 i,-3-3 i, 3-$ $3 i]$. These Asterisms at the first detection stage are centered at $h_{1} \times s_{i}$. Every possible combination is covered by these 16 Asterism circles. The size and the amount of overlap of these circles is determined by the number of transmit antennas, the magnitude of the elements of $H$ and the Hamming distance of the constellation.

If the received vector $R$ is inside the one or more circles it is possibly the ML solution. The algorithm then subtracts this possible solution from $R$ and determines whether modified $R$ is in one of the new Asterism circles centered at $h_{2} \times s_{i}$ and of radius $\left|h_{3}\right|$. This recursive process continues until all $n_{t}$ symbols are found. If there is more than one combination found, the combination with the lowest complex distance measurement is chosen to be the ML solution.

\section{A. Complexity}

The computational complexity of Asterism decoding for a Rayleigh fading environment was shown in [5] and is summarized in Table I.

\begin{tabular}{|c|c|c|c|}
\hline$n_{t}$ & Modulation & Asterism & ML \\
\hline 2 & 16QAM & 34 & 256 \\
3 & QPSK & 8 & 64 \\
3 & 16QAM & 220 & 4096 \\
4 & QPSK & 24 & 256 \\
4 & 16QAM & 2052 & 65536 \\
8 & QPSK & 2656 & 65536 \\
\hline
\end{tabular}

TABLE I

COMPLEXITY OF MAXIMUM LIKELIHOOD AND ASTERISM DECODING.

Table I compares the mean number of complex distance measurements (ML tests). It can be seen Asterism decoding has an approximate order of magnitude reduction in computational complexity compared to ML decoding. Also as the number of transmit antennas increases the greater the benefit of Asterism decoding is over ML decoding.

Although Asterism decoding's computational complexity is approximately an order of magnitude less that ML decoding, it can be seen from Table I that it is related to the number of transmit antennas and constellation size. For the system with $n_{t}=8$ and $Q P S K$ Asterism decoding would have higher complexity to that of the Sphere decoders system shown in Figure 1, making it far too complex for systems with a large number of transmit antennas.

Also, for systems with much larger number of receive antennas determining the optimal maximum ratio combining of [5] for Asterism decoding becomes more difficult due to the number of combinations that the received vector can be combined.

\section{COMbined Asterism AND SPHERE DECODER}

To reduce the complexity of Sphere decoding for $n_{t}>n_{r}$ we now propose using Asterism decoding to decode the first $n_{t}-n_{r}$ detected symbols. The Sphere decoder algorithm described in [4] and in Section III applies to a real system of equations and it chooses symbols from a real lattice, as opposed to complex symbols for Asterism decoding. To allow a combined Asterism and Sphere decoder we need to use complex Sphere decoder similar to [6], but make the adjustment of calculating a complex distance test to determine the points inside the sphere rather than the cosine algorithm of [6].

By using a sorted symbol detection order, i.e. detecting the symbols with the larger channel coefficients first and detecting the symbols with the smallest channel coefficients last, the Asterism decoder can detect the symbols with the largest channel coefficients, while the remaining symbols are detected by the Sphere decoder. At has has also been found that a sorted detection order has the additional benefit of reducing the complexity of the complex Sphere decoder. 


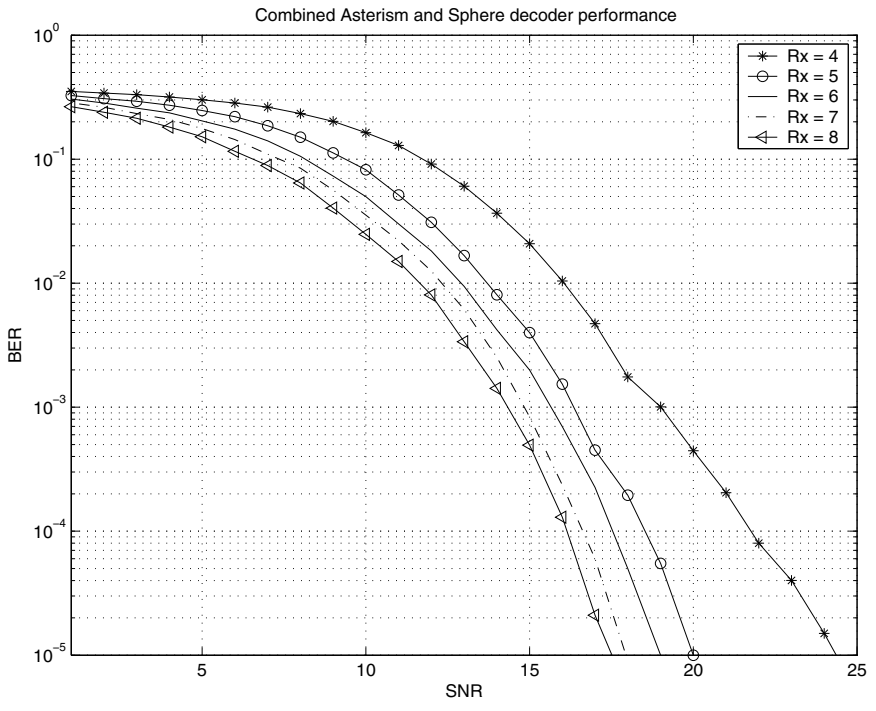

Fig. 3. Performance comparison of combined Asterism Sphere decoder for $n_{t}=8, n_{r}=4,5,6,7$ and 8 using $Q P S K$.

\section{Simulation Results}

In this section we provide simulation results, by Monte Carlo methods, to illustrate the performance of combined Asterism and Sphere decoding. Simulations indicate that there is no difference in the performance of combined Asterism and Sphere decoder, ML decoder and straight Sphere decoder. Figure 3 shows the performance of the combined Asterism and Sphere decoder for the system with $n_{t}=8$ using $Q P S K$ and $n_{r}=4,5,6,7$ and 8 .

The performance of the system with $n_{r}=4$ (approximately $10^{-3}$ at $19 \mathrm{~dB}$ ) is only $2.5 \mathrm{~dB}$ worse than the system with $n_{r}=5$. When $n_{r}=6$ the simulation show an additional $1 d B$ gain over the $n_{r}=5$ system, while when $n_{r}=7$ and 8 is only approximately $0.5 d B$ better than $n_{r}=6$ results. Giving an overall difference between $n_{r}=4$ and $n_{r}=8$ of less than $5 d B$ at a $B E R$ of $10^{-3}$.

Figure 4 shows the mean number of complex decoding stages which is a good representation of the complexity of a complex Sphere and Asterism combined Sphere decoder versus $S N R$ for a fixed and variable radius sizes.

It can be seen that from Figure 4 that the complexity of the combined Asterism and Sphere decoder is significantly less than the previously described complex Sphere decoder, this benefit is increases as $S N R$ decreases.

The starting radius of the results called 'large variable' is that of [4] which was stated as $2 n_{t} \sigma^{2}$ and the staring radius of the 'small variable' results was $\left(n_{t}-n_{r}\right) \sigma^{2}$. Choosing a smaller starting radius produces a lower complexity because the first $n_{t}-n_{r}$ symbols have been found by the Asterism decoder which does not use the noise variance to determine which symbols could be the ML solution.

Also of note is that smaller fixed radius has a lower computational complexity at low $S N R$ than the 'small variable' radius combined Asterism and Sphere decoding.

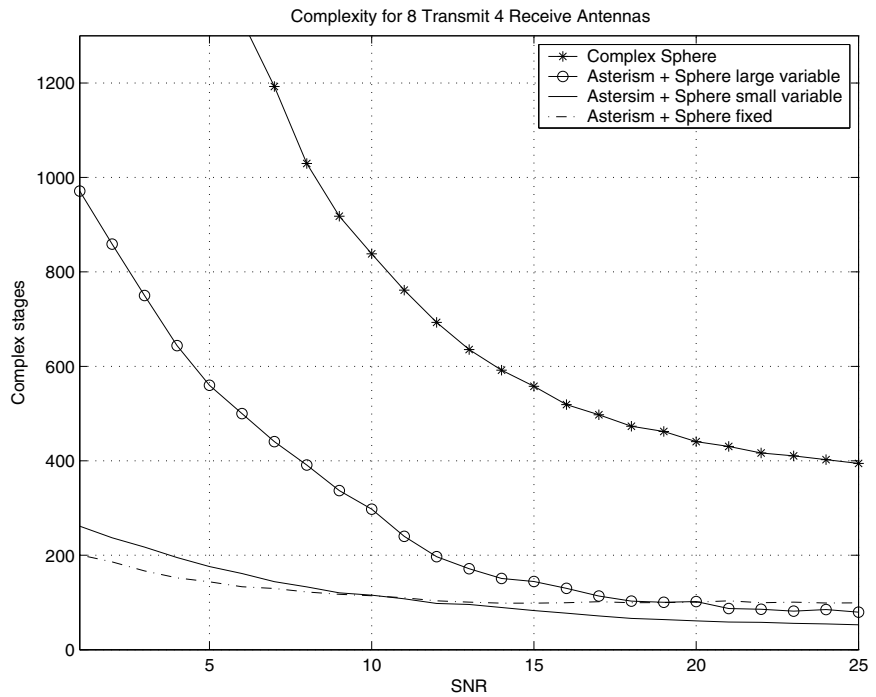

Fig. 4. Mean number of decoding stages of a complex and Asterism combined Sphere decoder versus $S N R$ for fixed and variable values of radius sizes.

\section{CONCLUSION}

This paper showed how Asterism decoding can be used in initial stages of a complex sphere decoder for asymmetric MIMO systems. It also shows how the computational complexity of using a combined Asterism and Sphere decoding is significantly reduced from that of Sphere decoding for all $S N R s$. It was also shown that for a system with 8 transmit antennas the performance loss by halving the number of receive antennas was less than $5 d B$. Reducing the size and cost of mobile terminals by reducing the number receive antennas is the main advantage of the combined Asterism and Sphere decoder as opposed to previously described MIMO decoders.

While systems where each user has more transmit antennas than receive antennas maybe unlikely, decoding may be utilized in reducing interference from neighboring cell/users or in a system that uses multipath fading as a multiple user access system.

\section{REFERENCES}

[1] E. Telatar, "Capacity of Multi-antenna Gaussian Channels", AT \& T Bell Labs, Murray Hill, NJ, Tech. Rep., 1995

[2] D. Garrett, L. Davis, G. Woodward, “ $19.2 \mathrm{Mbit} / \mathrm{s} 4 / \mathrm{spl}$ times/ 4 BLAST/MIMO detector with soft ML outputs", Electronics Letters , Volume: 39 Issue: 2 , 23 Jan. 2003.

[3] G.J. Foschini, "Layered Space-Time Architecture for Wireless Communications in a Fading Environment when using Multiple Antennas", Bell Labs Technical Journal, Autumn 1996.

[4] M. O. Damen, A. Chkeif, and J. C. Belfiore, "Lattice code decoder for space time codes", IEEE Communications Letters, May 2000.

[5] P. Conder and Tadeusz A. Wysocki, "Extending Asterism decoding to QAM and its complexity in Rician fading MIMO systems", Workshop on IT and Signal Processing 2004, Adelaide, Australia, 20-22 Dec 2004.

[6] Bertrand M. Hochwald, Stephan ten Brink, "Achieving Near-Capacity on a Multiple-Antenna Channel", IEEE Trans. on Communs, March 2003. 